\title{
Quantifying the Ordering of Adsorbed Proteins in Situ
}

\author{
Jie Wang, Sang-Ho Lee, Zhan Chen*
}

\section{Supporting Information}

\section{SFG}

We consider a laboratory fixed axis system $(x, y, z)$, where $z$ is parallel to the surface normal of the interface where protein molecules are adsorbed, and the incoming laser beams are in the $x z$ plane. Assuming that the interface with adsorbed protein molecules possesses azimuthal symmetry, seven independent elements in the second order nonlinear susceptibility would be nonzero. They are: $\chi_{z z z}, \chi_{z x x}=\chi_{z y y}, \chi_{x z x}=\chi_{y z y}$, $\chi_{x x z}=\chi_{y y z}, \chi_{x y z}=-\chi_{y x z}, \chi_{x z y}=-\chi_{y z x}$ and $\chi_{z x y}=-\chi_{z y x}$. The ratios among these tensor elements as well as their absolute values can be deduced from SFG spectra collected with different polarization combinations and from absolute intensity measurements. Such susceptibility element ratios and absolute values are related to molecular hyperpolarizability elements through average orientation. Usually SFG can provide more measurements than linear spectroscopic techniques. Therefore, SFG has the potential to provide a wealth of orientation and structural information of molecules and functional groups on surfaces and at interfaces, such as their orientation distribution and the absolute orientation direction.

In more detail, usually, SFG spectra can be fitted and analyzed by equation 1:

$$
I_{S F G} \propto\left|\chi_{\text {eff }}\right|^{2}=\left|\chi_{n r}+\sum_{q} \frac{A_{S F G, q}}{\omega_{2}-\omega_{q}+i \Gamma_{q}}\right|^{2}
$$


where $\chi_{n r}$ is the nonresonant background and $A_{S F G, q}$ is the amplitude of the qth vibrational mode. The molecular orientation and structural information of molecules at the interface can be obtained by relating the laboratory coordinate based susceptibility to the molecular coordinate based hyperpolarizability $\beta_{l m n}(l, m, n=a, b, c)$ through the Euler angles. The hyperpolarizability is an intrinsic property of a vibrational mode of a specific chemical group and their values can be calculated or measured. For the qth vibration mode, we have

$\chi_{i j k, q}^{(2)}=N \sum_{l, m, n}<(\hat{i} \cdot \hat{l})(\hat{j} \cdot \hat{m})(\hat{k} \cdot \hat{n})>\beta_{l m n, q}$

where $N$ is the density (or interfacial coverage) of the chemical groups and the brackets denote averaging over the molecular orientation distribution. In a typical visible-infrared SFG process, the hyperpolarizablity can be deduced from the infrared and Raman characteristics of a certain vibration mode through the following equation:

$\beta_{l m n, q} \propto \frac{\partial \alpha_{l m}^{*}}{\partial Q_{q}} \frac{\partial \mu_{n}}{\partial Q_{q}}$

where superscript “*” represents the complex conjugate, $\partial \mu_{n} / \partial Q_{q}$ and $\partial \alpha_{l m} / \partial Q_{q}$ are the infrared dipole and the Raman polarizability derivatives with respect to the normal coordinate of the qth vibrational mode, respectively, and $(l, m, n)$ are the molecular coordinate indices. Typically, SFG can only detect the modes which are both infrared and Raman active.

\section{Analysis of the Hyperpolarizability of $\alpha$-Helices}


Detailed analysis of symmetry operations of an infinitely long $\alpha$-helix (or a repeat unit, Figure S1) shows that its amide I vibrations are classified into three irreducible representations: $A, E_{1}$ and $E_{2} .(1-3)$ The A mode is a symmetric stretching mode parallel to the principal axis of the $\alpha$-helix, and the $E_{1}$ and $E_{2}$ modes are perpendicular to this principal axis. Because the $\mathrm{A}$ and $\mathrm{E}_{1}$ modes are both IR and Raman active, while the $\mathrm{E}_{2}$ mode is Raman active only, theoretically, only the A and $\mathrm{E}_{1}$ modes can be detected by SFG.

The IR transition dipole moments and Raman polarizability tensors of $\alpha$-helix amide I modes have been studied.(1-3) The SFG hyperpolarizarbility tensor of an $\alpha$-helix is a product of an IR transition dipole moment and a Raman polarizability tensor. It was reported that the IR transition dipole moments and Raman polarizability tensors of an $\alpha$ helix can be written in the following forms: $(2,3)$

IR transition dipolar moments:

A mode:

$$
\left[\frac{\partial \mu}{\partial Q^{r}}\right]\left(0^{\circ}\right)=\left(\begin{array}{l}
0 \\
0 \\
P_{r}^{c}\left(0^{\circ}\right) e^{i \delta_{A 0}}
\end{array}\right)
$$

$\mathrm{E}_{1}$ mode:

$$
\left[\frac{\partial \mu}{\partial Q^{r}}\right]\left(100^{\circ}\right)=\left(\begin{array}{l}
P_{r}\left(100^{\circ}\right) e^{i \delta_{E 0}} \\
P_{r}\left(100^{\circ}\right) e^{i\left(\delta_{E 0}+\frac{\pi}{2}\right)} \\
0
\end{array}\right)
$$




$$
\left[\frac{\partial \mu}{\partial Q^{r}}\right]\left(-100^{\circ}\right)=\left(\left[\frac{\partial \mu}{\partial Q^{r}}\right]\left(100^{\circ}\right)\right)^{*}=\left(\begin{array}{l}
P_{r}\left(100^{\circ}\right) e^{-i \delta_{E 0}} \\
P_{r}\left(100^{\circ}\right) e^{-i\left(\delta_{E 0}+\frac{\pi}{2}\right)} \\
0
\end{array}\right)
$$

Stokes Raman polarizability tensors:

A mode:

$$
\left[\frac{\partial \alpha}{\partial Q^{r}}\right]\left(0^{\circ}\right)=\left(\begin{array}{ccc}
\zeta_{r}^{a a}\left(0^{\circ}\right) e^{i \Theta_{A 0}} & 0 & 0 \\
0 & \zeta_{r}^{b b}\left(0^{\circ}\right) e^{i \Theta_{A 0}} & 0 \\
0 & 0 & \zeta_{r}^{c c}\left(0^{\circ}\right) e^{i \Theta_{A 0}}
\end{array}\right)
$$

(S7)

$$
\zeta_{r}^{a a}\left(0^{\circ}\right)=\zeta_{r}^{b b}\left(0^{\circ}\right)
$$

\section{(S8)}

$\mathrm{E}_{1}$ mode:

$$
\left[\frac{\partial \alpha}{\partial Q^{r}}\right]\left(100^{\circ}\right)=\left(\begin{array}{llc}
0 & 0 & \zeta_{r}^{a c}\left(100^{\circ}\right) e^{i \Theta_{E 0}} \\
0 & 0 & \zeta_{r}^{b c}\left(100^{\circ}\right) e^{i\left(\Theta_{0 E}+\frac{\pi}{2}\right)} \\
\zeta_{r}^{a c}\left(100^{\circ}\right) e^{i \Theta_{E 0}} & \zeta_{r}^{b c}\left(100^{\circ}\right) e^{i\left(\Theta_{E 0}+\frac{\pi}{2}\right)} & 0
\end{array}\right)
$$

(S9)

$$
\left[\frac{\partial \alpha}{\partial Q^{r}}\right]\left(-100^{\circ}\right)=\left(\left[\frac{\partial \alpha}{\partial Q^{r}}\right]\left(100^{\circ}\right)\right)^{*}=\left(\begin{array}{ccc}
0 & 0 & \zeta_{r}^{a c}\left(100^{\circ}\right) e^{-i \Theta_{E 0}} \\
0 & 0 & \zeta_{r}^{b c}\left(100^{\circ}\right) e^{-i\left(\Theta_{E 0}+\frac{\pi}{2}\right)} \\
\zeta_{r}^{a c}\left(100^{\circ}\right) e^{-i \Theta_{E 0}} & \zeta_{r}^{b c}\left(100^{\circ}\right) e^{-i\left(\Theta_{E 0}+\frac{\pi}{2}\right)} & 0
\end{array}\right)
$$

(S10)

$$
\zeta_{r}^{a c}\left(100^{\circ}\right)=\zeta_{r}^{b c}\left(100^{\circ}\right)
$$


where $0^{\circ}$ and $\pm 100^{\circ}$ are the phase differences for the $\mathrm{A}$ and $\mathrm{E}_{1}$ mode vibrations respectively, between adjacent $\mathrm{C}=\mathrm{O}$ stretching groups along the peptide chain.(2,3) Intrinsic phase terms $\delta_{A 0}, \delta_{E 0}, \Theta_{A 0}$ and $\Theta_{E 0}$ are also included here for more generality.

The Raman polarizability tensor elements in equations S7-S10 are for Stokes Raman scattering, while the Raman polarizability elements used to calculate SFG hyperpolarizability tensor elements should be the tensor elements of anti-Stokes Raman scattering (the complex conjugate values of the Raman tensors in equations S7-S10). Therefore we have the following nonzero SFG hyperpolarizability tensor elements for the $\mathrm{A}$ and $\mathrm{E}_{1}$ modes:

A mode $\left(0^{\circ}\right)$ :

$\beta_{a a c}=\beta_{b b c} \propto \zeta_{r}^{a a}\left(0^{\circ}\right) P_{r}^{c}\left(0^{\circ}\right) e^{i\left(\delta_{A 0}-\Theta_{A 0}\right)}$

$\beta_{c c c} \propto \zeta_{r}^{c c}\left(0^{\circ}\right) P_{r}^{c}\left(0^{\circ}\right) e^{i\left(\delta_{A 0}-\Theta_{A 0}\right)}$

$\mathrm{E}_{1}$ mode $\left( \pm 100^{\circ}\right)$ :

$\mathrm{E}_{1}$ mode is a two-fold degenerate vibration in a plane perpendicular to the helix principal axis: one corresponds to the phase difference $100^{\circ}$ and the other corresponds to the phase difference $-100^{\circ}$ between adjacent peptide units. Since equations S6, S7, S9 and S10 are expressed based on a one-dimensional irreducible representation, the SFG hyperpolarizability tensor elements for the $\mathrm{E}_{1}$ mode are given by:

$$
\beta_{l m n, q} \propto \frac{\partial \alpha_{m l}^{*}}{\partial Q_{q}}\left(100^{\circ}\right) \frac{\partial \mu_{n}}{\partial Q_{q}}\left(100^{\circ}\right)+\frac{\partial \alpha_{m l}^{*}}{\partial Q_{q}}\left(-100^{\circ}\right) \frac{\partial \mu_{n}}{\partial Q_{q}}\left(-100^{\circ}\right)
$$

Therefore we have: 


$$
\begin{aligned}
& \beta_{a c a}=\beta_{c a a}=\beta_{b c b}=\beta_{c b b} \propto P_{r}\left(100^{\circ}\right) \zeta_{r}\left(100^{\circ}\right)\left[e^{i\left(\delta_{0}-\Theta_{0}\right)}+e^{-i\left(\delta_{0}-\Theta_{0}\right)}\right] \\
& \beta_{a c b}=\beta_{c a b} \propto P_{r}\left(100^{\circ}\right) \zeta_{r}\left(100^{\circ}\right)\left[e^{i\left(\delta_{0}-\Theta_{0}+\frac{\pi}{2}\right)}+e^{-i\left(\delta_{0}-\Theta_{0}+\frac{\pi}{2}\right)}\right] \\
& \beta_{b c a}=\beta_{c b a} \propto P_{r}\left(100^{\circ}\right) \zeta_{r}\left(100^{\circ}\right)\left[e^{i\left(\delta_{0}-\Theta_{0}-\frac{\pi}{2}\right)}+e^{-i\left(\delta_{0}-\Theta_{0}-\frac{\pi}{2}\right)}\right]
\end{aligned}
$$

The SFG process discussed here can be treated as an IR adsorption plus an inphase anti-Stokes Raman scattering; we can assume: $\delta_{A 0}=\Theta_{A 0}$ and $\delta_{E 0}=\Theta_{E 0}$. Therefore equation 14 can be further simplified to

$$
\begin{aligned}
& \beta_{a c a}=\beta_{c a a}=\beta_{b c b}=\beta_{c b b} \propto 2 P_{r}\left(100^{\circ}\right) \zeta_{r}\left(100^{\circ}\right) \\
& \beta_{a c b}=\beta_{c a b}=\beta_{b c a}=\beta_{c b a}=0
\end{aligned}
$$

It is obvious that there are only seven nonzero hyperpolarizability tensor elements remaining for an $\alpha$-helix: $\beta_{a a c}=\beta_{b b c}$ and $\beta_{c c c}$ (for A mode); $\beta_{a c a}=\beta_{c a a}=\beta_{b c b}=\beta_{c b b}$ (for $E_{1}$ mode). The above results can also be applied to one repeat unit of an $\alpha$-helix. In this paper, our discussion is focused on one repeat unit rather than an infinitely long helix.

\section{Polarized ATR-FTIR Data Analysis}

Assuming the surface for protein adsorption is the $x y$ plane, and the input light is in the $x z$ plane, the IR absorbance of the $q$ th vibrational mode in the ATR-FTIR spectra can be written as (4)

$W_{I R, q} \propto<\left(\mathbf{M}_{q} \cdot \mathbf{E}\right)^{2}>\propto<M_{q, x}^{2}>E_{x}^{2}+<M_{q, y}^{2}>E_{y}^{2}+<M_{q, z}^{2}>E_{z}^{2}$

Where $\mathbf{M}_{q}=\left(M_{q, x}, M_{q, y}, M_{q, z}\right)$ is the transition moment of the $q$ th vibrational mode in the laboratory fixed coordinate system. $\mathbf{E}=\left(E_{x}, E_{y}, E_{z}\right)$ is the electric field vector of the infrared beam in the sample. An angular bracket indicates an average over the 
orientational distribution in the sample. If the AIR-FTIR spectrum is collected using s or p polarized light, we have $(4,5)$

$W_{I R, q}^{S} \propto<M_{q, y}^{2}>E_{y}^{2}$

$W_{I R, q}^{p} \propto<M_{q, x}^{2}>E_{x}^{2}+<M_{q, \tau}^{2}>E_{z}^{2}$

Here, $W_{I R, q}^{S}$ and $W_{I R, q}^{P}$ are IR absorbance collected using $\mathrm{s}$ and $\mathrm{p}$ polarized light, respectively. When such data are acquired, orientation information of the transition dipole can be deduced.

Observed ATR-FTIR spectra have been extensively fitted in the literature with Lorentzian functions:

$W_{I R} \propto \sum_{q}\left|\operatorname{img}\left(\frac{A_{I R, q}}{\omega_{2}-\omega_{q}+i \Gamma_{q}}\right)\right|^{2}$

where $A_{I R, q}$ is the amplitude of the $q$ th vibrational mode, $\Gamma_{q}$ is the band width parameter and $\omega_{q}$ is the center frequency. The actual line shape of the amide I spectra can be more complicated, but here we decide to use Lorentzian function for fitting. The spectra were also fitted by using Gaussian functions as a comparison (not shown) and we found that fitting results are similar. Based on the coordinate systems defined in Figure S1, the transition dipole moments of an $\alpha$ helix can be written as $(4,5)$

$$
\begin{aligned}
& <M_{x}^{2}>=<M_{y}^{2}>\propto \int_{0}^{\pi}\left[\left(1+\cos ^{2} \theta\right) \sin ^{2} \Theta+2 \sin ^{2} \theta \cos ^{2} \Theta\right] f(\theta) \sin \theta d \theta \\
& <M_{z}^{2}>\propto \int_{0}^{\pi} 2\left(\sin ^{2} \theta \sin ^{2} \Theta+2 \cos ^{2} \theta \cos ^{2} \Theta\right] f(\theta) \sin \theta d \theta
\end{aligned}
$$


where $\Theta$ is the orientation angle of the transition dipole moment of a single $\mathrm{C}=\mathrm{O}$ bond ( $\mathrm{N}-\mathrm{H}$ bond for amide II) relative to the axis of the $\alpha$-helix. As defined in Figure S1, $\theta$ is the orientation angle between the axis of the $\alpha$-helix and the sample surface normal. $f(\theta)$ is the orientation distribution function. Therefore orientation information can be deduced by measuring $W_{I R, q}^{S}$ and $W_{I R, q}^{P}$.

\section{Evaluation of the Local Optical Fields in Polarized ATR-FTIR Measurements.}

When we deduce orientation information of an $\alpha$ helical structure at the interface using polarized ATR-FTIR (e.g., to compare absorbance from s polarized beam to p polarized beam), it is necessary to have local field information for $E_{x}, E_{y}$, and $E_{z}$. We can calculate such local field information of protein molecules at the PS/protein solution interface using the refractive indices of the ATR crystal, PS, protein layer, and protein solution. The accuracy of these local field calculations highly depends upon the accuracies of such refractive indices. Additionally, the polymer film thickness may also dramatically affect the local optical fields. We confirmed our calculations of local optical fields by doing experiments using a polymer film. If a thin layer of molecules with a random orientation distribution is deposited on the PS surface, the difference of IR absorbance between s and p polarizations of this layer is only due to the differences in the local optical fields. We designed a simple experiment to evaluate our calculations. Two PS films with different thicknesses were deposited on the ATR crystal. The thickness of one film is close to the thickness used in the protein adsorption experiment and the other film is a little bit thicker. The difference in the film thicknesses is used to represent an adsorbed layer of molecules on the thinner film. By assuming that polymer molecules have a random orientation distribution in the bulk, the IR absorbance differences between 
the two films with s and p polarizations can be used to evaluate the local field differences on the thin film surface. For our experimental conditions, the absorption in PBS solution with the p polarization is about 1.55 times higher than with the s polarization, which is very close to the calculated ratio (1.5). It indicates that our calculated results can be used as a valid approximation. If the adsorbed proteins have a random orientation distribution, the ATR-FTIR absorbance measured with p polarized light should be about 1.5 times higher than that measured with the s polarized beam.

\section{Figure Captions}

Figure S1. Euler angles between a laboratory fixed coordinate system (x, y, z) and a molecular fixed coordinate system (a, b, c) for an $\alpha$-helix.

\section{References}

1. Higgs, P. W. (1953) Proc. R. Soc. London A 133, 472-485.

2. Fanconi, B.; Tomlinson, B., Nafie, L. A., Small, W., Peticolas, W. L. (1969), J. Chem. Phys. 51, 3993-4005.

3. Lee, S.-H. \& Krimm, S. (1998) Chem. Phys. 230, 277-295

4. Marsh, D. (1997) Biophys. J. 72, 2710-2718

5. Marsh, D., Müller, M. \& Schmitt F.-J. (2000) Biophys. J. 78, 2499-2510. 


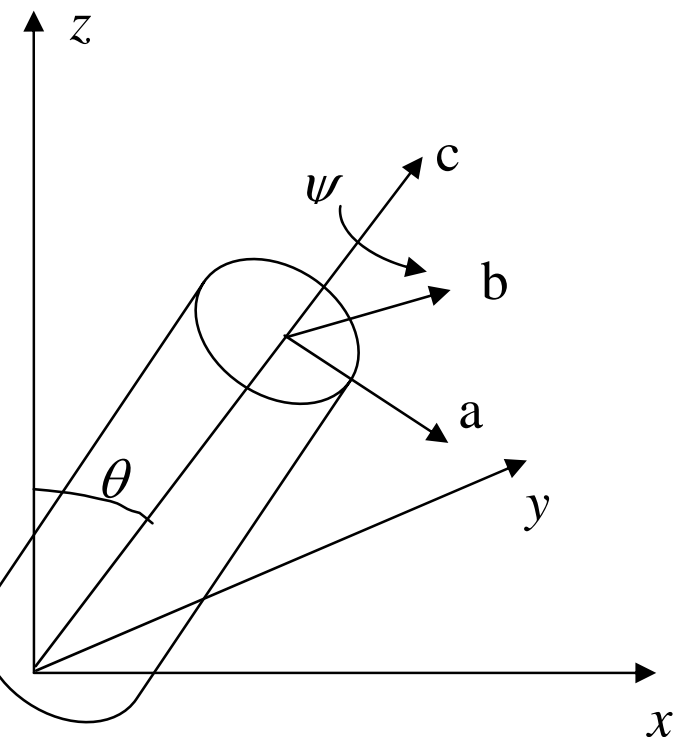

Figure S1 